\title{
Fabrication of Depth-controllable Nanochannel with Polymer Nanoassembled Films Using Atomic Force Microscopy Lithography
}

\author{
Takeshi Mikayama, Toshio SuZuki, Jun Matsui, and Tokuji MiYashita ${ }^{\dagger}$ \\ Institute of Multidisciplinary Research for Advanced Materials, Tohoku University, \\ 2-1-1 Katahira, Aobaku, Sendai 980-8577, Japan
}

(Received July 4, 2005; Accepted July 14, 2005; Published November 15, 2005)

\begin{abstract}
Fabrication of a nanochannel consisting of poly( $N$-neopentylmethacrylamide) Langmuir-Blodgett film (polymer nanosheet) utilizing atomic force microscopy (AFM) lithography is demonstrated. We obtained the nanochannel with $1-20 \mathrm{~nm}$ depth and $150-280 \mathrm{~nm}$ width. The channel surface is highly homogenous which is an advantage for covering the channel tightly without leaking point. The depth of the channel increases linearly with increasing number of layers, which indicates that the channel depth is controllable in a nanometer level. The width of the nanochannel is independent with the thickness of the film. [DOI 10.1295/polymj.37.854]

KEY WORDS Langmuir-Blodgett Film / Polymer Nanosheet / Nanochannel / Poly $(N$-neopentylmethacrylamide) / Atomic Force Microscopy / AFM Lithography /
\end{abstract}

Microfluidic devices have wide application in chemistry and biology for manipulating samples in small quantities, fast, high-resolution and low cost analysis and synthesis. ${ }^{1}$ The devices consist of several channels and a cover plate. In the emerging of nanobiotechnology, further downsizing the fluidic channel to the nanometer scale with well-controlled dimensions is desired. Microfabrication of glass and silicon substrate by photolithography has been used most commonly to prepare microchannels. ${ }^{2,3}$ However they are expensive to produce and poorly suited for introducing specific chemical functionalities into each channels. Recently much interest has been paid to construct microfluidic devices using soft materials such as polymers because of low cost fabrication. ${ }^{4}$ Poly(dimethylsiloxane) (PDMS) is the most widely used material for construction of microfluidic device. For fabricating channels onto PDMS, micro or nanoimprinting technique has been commonly used. These contact printing techniques afford easy and low cost fabrication. ${ }^{5}$ In the nanoimprinting technique, it is easy to control the depth with several tens of nanometer resolution by a simple relationship involving the initial polymer layer thickness and the mold pattern configuration. However, to put functionality to the PDMS and/or to change surface properties, plasma treatment is required. Moreover, it is very difficult to control the channel scale in molecular level.

We have reported that several $N$-alkylacrylamide polymers form stable monolayer at the air/water interface. ${ }^{6-8}$ The polymer monolayer is particularly attractive as materials for microfluidic device. The film thickness is easily controllable with nanometer resolu- tion by changing the deposition number of layers. Thus the channel depth is controllable in nanometer resolution. Moreover, the surface of the nanosheet is molecularly smooth. The smooth surface affords us to cover the channel without leaking point. The integration of electronic or fluorescent probe molecules can be incorporated into polymer nanosheet by copolymerization or chemical reaction.

We have reported the pattering of polymer nanosheet using photolithography technique. ${ }^{9}$ However, conventional lithography causes irradiation-induced damage to photo-responsive groups which are incorporated in polymer as a fluorescent probe. In addition, a high-vacuum environment and solvents for developing the photopattern are often required. On the other hand atomic force microscope (AFM) is a powerful tool not only for surface observation but also for mechanical nano-pattering of the soft materials. ${ }^{10}$ Furthermore AFM lithography can be carried out in air without light and any solvent, which is advantage for creating soft nanochannel with fluorescent probe. In this paper, we demonstrate the fabrication of nanochannel with polymer nanosheet by AFM lithography (Figure 1).

\section{EXPERIMENTAL}

The preparation of poly( $N$-neopentylmethacrylamide) (pnPMA) is described in the previous paper. ${ }^{11}$ Molecular weight and polydispersity value were $2.6 \times$ $10^{4}$ and 1.3 , respectively. The depositions of the monolayers were carried out with a computer-controlled Langmuir trough (FSD-110 from USI Inc, Japan) at

${ }^{\dagger}$ To whom correspondence should be addressed (Tel: +81-22-217-5637, FAX: +81-22-217-5642, E-mail: miya@ tagen.tohoku.ac.jp). 


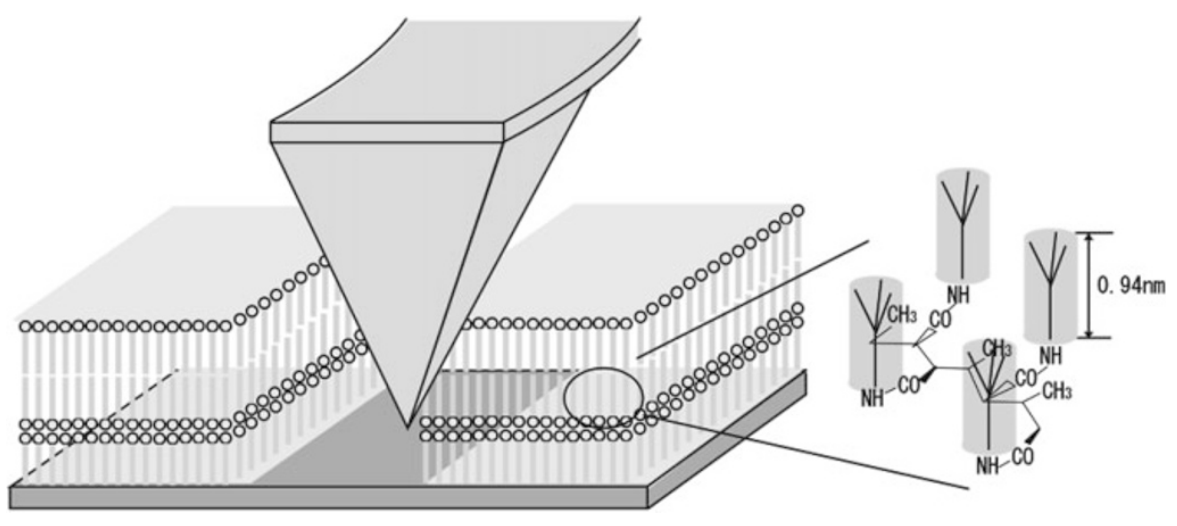

Figure 1. Fabrication of nanochannel with polymer nanosheets using AFM lithography.

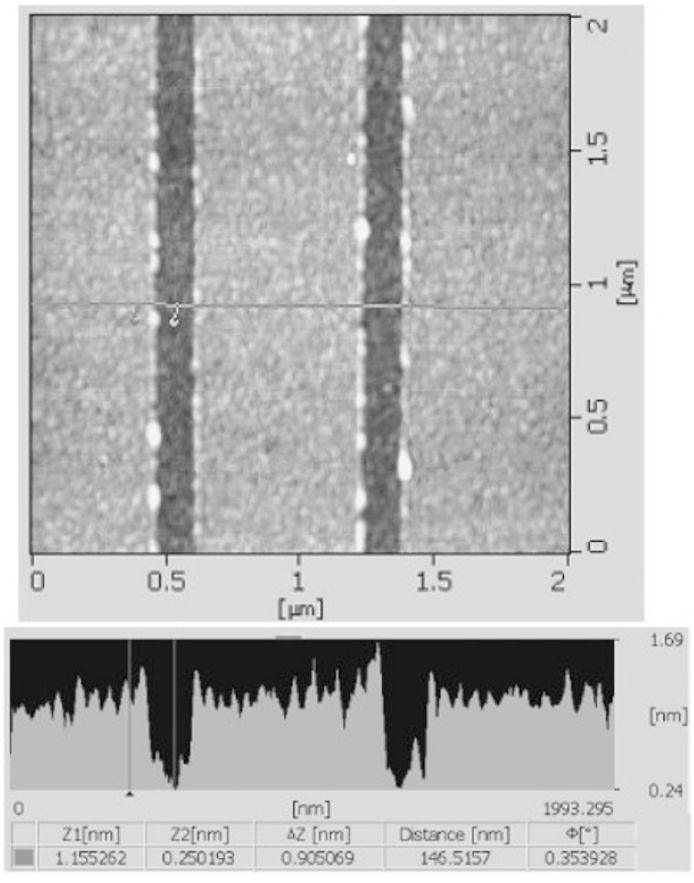

(a)

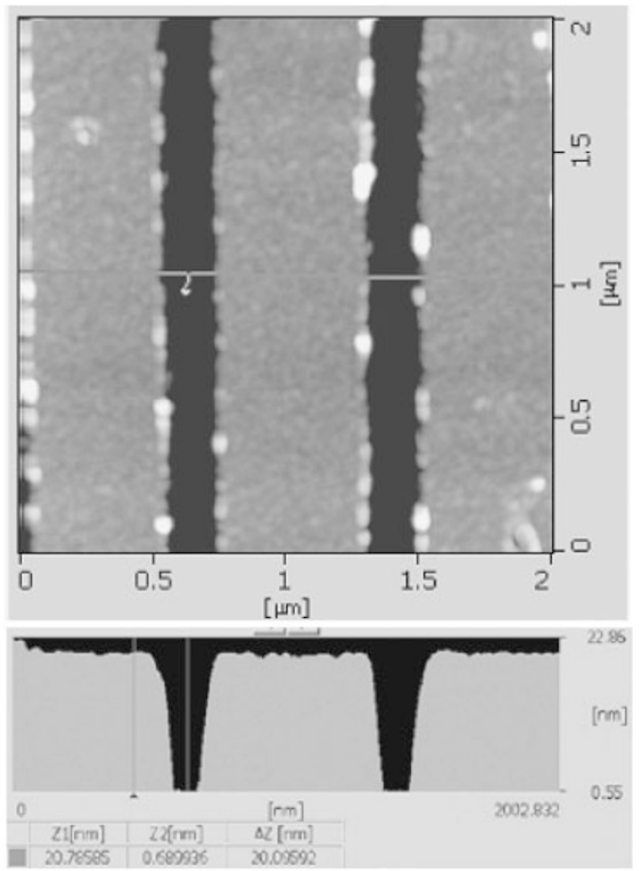

(b)

Figure 2. AFM image and corresponding cursor profile of pnPMA monolayer (a) and pnPMA 21 layers (b) after AFM lithography.

$15^{\circ} \mathrm{C}$. Distilled and deionized water (CPW-101, Advantec) was used for the subphase. Chloroform was used as solvent for spreading monolayer on the water surface. A silicon wafer was cleaned by UV-ozone treatment to make the surface hydrophilic and used as a substrate. The monolayer on the water surface was transferred onto the hydrophilic silicon wafer using the vertical dipping method under a surface pressure of $20 \mathrm{mN} / \mathrm{m}$. A commercial AFM (SPA-400 and SPI-3800 from SII Nanotechnology, Inc. Japan) was used in all AFM experiments. Mechanical patterning of nanochannel was carried out by scratching the polymer nanosheets using the AFM cantilever with high spring constant at room temperature in the air condition. The silicon cantilever with $k=40 \mathrm{~N} / \mathrm{m}$ and small tip radius curvature (less than $10 \mathrm{~nm}$ )
(SI-DF40 from OLYMPUS Inc., Japan) was used in contact mode. The cantilever was moved with the speed of $1 \mathrm{~mm} / \mathrm{s}$ and the applied force was $2-4 \mu \mathrm{N}$ to draw patterns onto the polymer nanosheet. After the AFM lithography, surface morphology was observed using tapping mode in air condition. The spring constant of the cantilever used for tapping mode is $k=20 \mathrm{~N} / \mathrm{m}$ (SI-DF 20 from OLYMPUS Inc.).

\section{RESULTS AND DISCUSSION}

A chloroform solution of pnPMA was spread onto a water surface to form the polymer monolayer at the air/water interface. The surface pressure $(\pi)$-surface area $(A)$ isotherms of the polymer, which were already studied clearly in the previous paper, ${ }^{11}$ shows steep 
rise with decreasing surface area. This indicates that a stable monolayer (polymer nanosheet) is formed at the air/water interface. The pnPMA-nanosheet was transferred onto silicon substrate using the LB technique. The transfer ratios were 1.0 in both downward and upward strokes resulting in the Y-type LB film. The surface morphology of the nanosheet was observed by AFM using tapping mode. The transferred film shows no pinhole in the observed area (several $\mu \mathrm{m}^{2}$ ). Moreover the surface roughness of pnPMA nanosheet is less than $0.6 \mathrm{~nm}$. These results indicate that highly homogenous and smooth surface onto solid substrates was formed. The pinhole free nanosheet is resulted from the hydrogen-bonding network between amide bonds in the pnPMA-nanosheet. Further, the surface shows the amorphous nature, which is advantage in high tolerance against mechanical bending.

First, the pnPMA monolayer deposited onto silicon was scratched using AFM cantilever at room temperature in air. The force to scratch the nanosheet is $2-4 \mu \mathrm{N}$ and the speed is $1 \mathrm{~mm} / \mathrm{s}$. After the scratching, the surface was observed by AFM using tapping mode (Figure 2a). The channel depth is $0.9 \pm 0.3 \mathrm{~nm}$, which correspond to the monolayer thickness of pnPMA $(0.94 \mathrm{~nm})$-nanosheet determined from XRD analyses. ${ }^{11}$ Thus the short-branched alkyl substituents in monolayer are oriented perpendicular to the silicon substrate. The width of nanochannel is $140 \pm 10 \mathrm{~nm}$, which is much larger than the tip radius curvature $(20 \mathrm{~nm})$ used in the scratching. Jahromi et al. also reported the width of the channel around $200 \mathrm{~nm}$ using AFM for scratching a poly(methyl methacrylate) cast film. ${ }^{12}$ The larger channel width is resulted from lateral interaction between the polymer chains. A few amount of polymer chains are removed together by the AFM tip due to lateral force such as van der Waals force between polymer chains and hydrogen bonding between the amide groups in the polymer main chains.

Next we tried to scratch the multilayers. The AFM image and cross-section profile show that a few amount of polymer chains move orthogonal to the scan direction and accumulate on the both sides of nanochannel. Large grains were observed at the edge of the channel, which is the point of turning the scan direction (Figure 3). Thus most of the shaved polymer adsorb on the tip apex and move with the tip. When the amount of adsorbed polymer become large, they move from the tip to the film surface at the turn-round point of scan and remain a grain of polymer.

To demonstrate the depth control of the nanochannel, multilayer film with different number of layers was shaved by the same technique. Figure $2 b$ shows the 21 layers AFM image after shaving the film by AFM cantilever. The channel depth is $20.1 \mathrm{~nm}$, which corresponds to the thickness of 21 layers of pnPMA

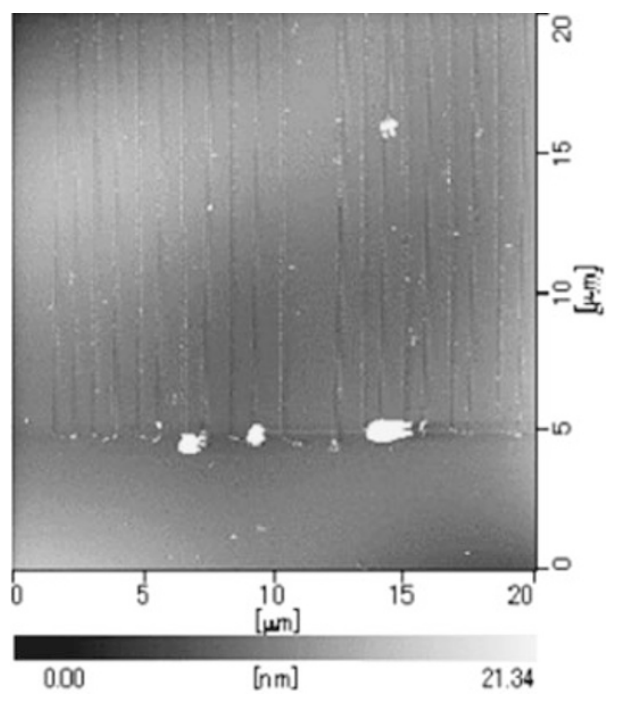

Figure 3. AFM image of pnPMA monolayer in wide area (20 $\mu \mathrm{m}$ scan) after AFM lithography.

calculated from XRD measurement. Thus the shortbranched alkyl substituents in each layer are oriented perpendicular to the substrate and the tip apex reaches the Si substrate surface while scratching. Moreover the multilayer shows a highly homogenous surface with surface roughness of $1.2 \mathrm{~nm}$, which is advantage for good sealing after capping cover plate on nanochannel. The width of nanochannel with $150 \pm 10$ $\mathrm{nm}$ is almost same as that after scratching of pnPMA monolayer. The nanochannel depth is easily controllable by changing the deposition number of the layers (Figure 4). The depth increases linearly with increasing number of layer and correspond to the calculated layer thickness due to a regular deposition of the pnPMA monolayer. This means we can control the nanochannel thickness in nanometer resolution. Interestingly the width of the nanochannel fluctuates between 150 and $280 \mathrm{~nm}$ with different number of layers and it is independent of the film thickness. This means the width of the nanochannel depends on lateral force between the polymer chains such as hydrogen bonding and van der Waals force. To control the channel width it will be necessary to control the experimental condition such as temperature, humidity. It is expected that the width of nanochannel can be controlled by changing the shaving temperature because the lateral forces depend on temperature.

\section{CONCLUSIONS}

We fabricated nanochannel consisting of polymer nanosheet using AFM lithography. The channel surface is highly homogenous which make us easy to cap the nanochannel with good sealing. The depth of the channel increases linearly with increasing number of 


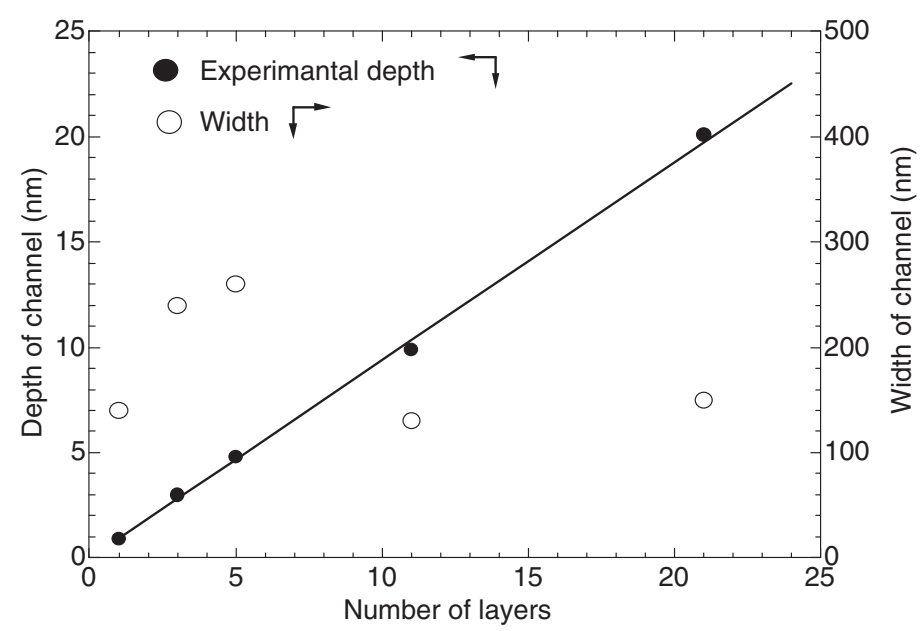

Figure 4. Plots of nanochannel depth (filled circle) and width (open circle) with different deposition number of layers determined from AFM measurements. Calculated layer thickness due to a regular deposition of pnPMA monolayer is also plotted (solid line).

layers. The width of the nanochannel is independent with the thickness of the film, which indicate the depth is controllable in nanometer resolution.

Acknowledgment. This work is partially supported by a Grant-in-Aid for Scientific Research "No. 17105006". The authors thank technical laboratory in Hybrid Nano-Materials Research Center (HyNam Center) for AFM measurements.

\section{REFERENCES}

1. T. Thorsen, S. J. Maerkl, and S. R. Quake, Science, 298, 580 (2002)

2. A. G. Hadd, D. E. Raymond, J. W. Halliwell, S. C. Jacobson, and J. M. Ramsey, Anal. Chem., 69, 3407 (1997).

3. M. A. Burns, B. N. Johnson, S. N. Brahmasandra, K. Handique, J. R. Webster, M. Krishnan, T. S. Sammarco,
P. M. Man, D. Jones, D. Heldsinger, C. H. Mastrangelo, and D. T. Burke, Science, 282, 484 (1998).

4. S. Hu, X. Ren, M. Bachman, C. E. Sims, G. P. Li, and N. L. Allbritton, Langmuir, 20, 5569 (2004).

5. L. J. Guo, X. Cheng, and C.-F. Chou, Nano Lett., 4, 69 (2004).

6. T. Miyashita, Prog. Polym. Sci., 18, 263 (1993).

7. A. Aoki, Y. Abe, and T. Miyashita, Langmuir, 15, 1463 (1999).

8. J. Matsui, M. Mitsuishi, A. Aoki, and T. Miyashita, Angew. Chem., Int. Ed., 42, 2272 (2003).

9. A. Aoki and T. Miyashita, Adv. Mater., 9, 361 (1997).

10. G.-Y. Liu, S. Xu, and Y. Qian, Acc. Chem. Res., 33, 457 (2000).

11. Y. Guo, M. Mitsuishi, and T. Miyashita, Macromolecules, 34, 3548 (2001).

12. S. Jahromi, J. Dijkstra, E. Vegte, and B. Mostert, ChemPhysChem, 8, 693 (2002). 